\title{
Sensory integration of apparent motion speed and vibration magnitude
}

\author{
Matjaž Ogrinc ${ }^{1,2}$, Ildar Farkhatdinov ${ }^{3,1}$, Rich Walker ${ }^{2}$ and Etienne Burdet ${ }^{1}$
}

\begin{abstract}
Tactile apparent motion can display directional information in an intuitive way. It can for example be used to give directions to visually impaired individuals, or for waypoint navigation while cycling on busy streets, when vision or audition should not be loaded further. However, although humans can detect very short tactile patterns, discriminating between similar motion speeds has been shown to be difficult. Here we develop and investigate a method where the speed of tactile apparent motion around the user's wrist is coupled with vibration magnitude. This redundant coupling is used to produce tactile patterns from slow\&weak to fast\&strong. We compared the just noticeable difference (JND) of the coupled and the individual variables. The results show that the perception of the coupled variable can be characterised by JND smaller than JNDs of the individual variables. This allowed us to create short tactile pattens (tactons) for display of direction and speed, which can be distinguished significantly better than tactons based on motion alone. Additionally, most subjects were also able to identify the coupled-variable tactons better than the magnitude-based tactons.
\end{abstract}

Index Terms—vibrotactile, tactons, apparent motion, JND, wrist, wearable

\section{INTRODUCTION}

Tactons (also called haptic icons [1] or tactile melodies [2]) are abstract haptic messages that can employ variation of different physical parameters of vibrotactile stimulation, including waveform, magnitude, frequency, rhythm and location [3]. The simplest example of tactons can be found in our everyday lives; a smartphone can buzz in different temporal patterns to let us distinguish between a calendar event and an incoming text message. Conversely, attempts have been made to deploy sets as large as 84 tactons [4], challenging the human perceptual and associative capacity. Therefore, a tacton should encode the message in an intuitive way to ease the learning of associations. For instance, [5] employed metaphors based on user requirements to design tactile cues for spatial navigation.

Tactons commonly employ arrays of spatially distributed tactile stimulators and are used in spatial navigation and orientation tasks [5]-[10]. Tactile arrays can be activated in spatio-temporal patterns to create an illusion of apparent tactile motion. Apparent motion can suggest the user a change of direction to reach a point of interest and also provide information about various vector quantities such as motion, force and torque.

In the case of tactile apparent motion, the presented information can be encoded by the motion speed and direction of travel. To maximise the information transfer of a set of such tactons, the changes in speed must be clearly distinguishable and the vibration patterns short. Though using slow motion speeds may increase the number of distinct tactons, it also increases the minimum stimulation time. Therefore, our aim is to design motion based tactons that are fast (thus only require a short stimulation duration) but clearly distinguishable.

\footnotetext{
${ }^{1}$ Department of Bioengineering, Imperial College of Science, Technology and Medicine, London SW72AZ, UK. E-mail: e.burdet@imperial.ac.uk

${ }^{2}$ Shadow Robot Company, London N11LX, UK

${ }^{3}$ School of Electronic Engineering and Computer Science, Queen Mary University of London, London E14NS, UK
}

Previous work has shown that humans have difficulties in absolute ranking of the speed of apparent motion [11]. To overcome this limitation, we propose a novel stimulation method where the speed of apparent motion around the wrist is coupled with vibration magnitude. This coupling produces tactile motions that range from slow and weak to fast and strong. As the central nervous system seems to combine sensory information in a fashion that is similar to the maximum-likelihood integrator [12], we hypothesised that the proposed coupling will improve the users' ability to discriminate between similar motion speeds, which is investigated in the present work. First, we evaluated the maximum speed of apparent motion that can be achieved for the case of forearm stimulation using inertial (eccentric mass) motors. Then, we experimentally estimated the just noticeable difference (JND) of apparent motion speed, vibration magnitude, and the coupled modulation. Finally, we designed sets of tactons for each modulation type based on the estimated JND and evaluated the participants' ability to distinguish them using a rating task.

In the next section we present the relevant background and describe the proposed stimulation technique. We explain our interface design choices with respect to previous work on similar feedback approaches. Sections 3 and 4 report on two two-alternative forced choice experiments to determine the maximum realisable speed of apparent motion and to estimate the JND of the proposed stimulation approach. Finally, the rating task experiment, carried out in order to investigate the absolute discriminability, is presented and discussed in section 5 .

\section{Design of tactile stimulation}

\subsection{Just noticeable difference in tactile perception}

The just noticeable difference $\triangle I_{\mathrm{JND}}$ is the change of intensity of some physical variable $I$, such as vibration frequency, acceleration, duration, or motion speed, that can be detected 

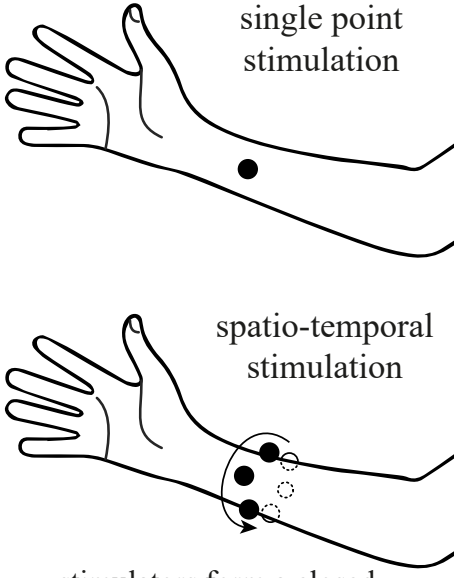

stimulators form a closed loop around the forearm magnitude $(M)$ modulation

(frequency, amplitude)

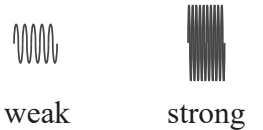

motion speed $(S)$

modulation

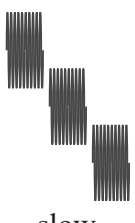

slow

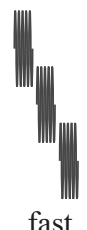

fast pulse width modulation

(temporal)

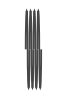

short long

motion speed \& magnitude

$(S \& M)$ modulation

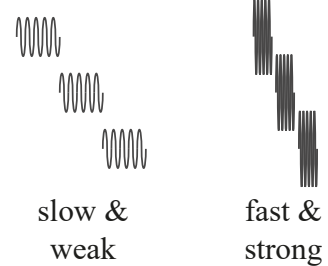

Fig. 1: Types of tactile stimulation provided to the forearm depicted as vibration amplitude against time. Using a single point stimulation (top), vibration frequency, amplitude and temporal parameters can be varied to create a set of cues. A tactile array (bottom) can employ combination of encodings to produce spatio-temporal and magnitude-spatio-temporal cues.

at least half the time. For many sensory modalities, the perception follows Weber's law according to which $w=\frac{\triangle I_{\mathrm{JND}}}{I}$ (Weber fraction) is independent on $I$ [13]. It has been shown that the law applies to vibration magnitude [14], [15], frequency [16], [17] and speed of apparent motion [18]. In 1860, Weber's law has been extended by Fechner to explain the relationship between the stimulus intensity and perceived magnitude of a stimulus. Steven's law (1957) has since been found to better quantify the intensity-perceived magnitude curves for a number of sensory phenomena [19]. However, in this work we are not concerned with perceived absolute magnitude, but with the discrimination and ranking of similar intensities, in particular apparent motion speeds. In our design, the intensities of tactile stimuli, whether it is vibration magnitude or motion speed, are separated by the JND.

Choi et al. suggested that at least 20\%-30\% difference in vibration amplitude or frequency is necessary for robust discrimination between vibrotactile stimuli [14]. However, humans cannot discriminate between the changes in frequency and magnitude well. In fact, it has been shown that a change in either frequency or amplitude of stimulation can cause an equivalent change in perceived intensity [20], [21]. This limitation should be considered when designing tactile patterns based on independent control of frequency and vibration amplitude. The most common DC inertial actuators used for tactile stimulation, a sub-type of which is also known as pancake or coin motors, do not allow their independent control. Instead, both frequency and amplitude increase with the voltage applied to the motor. \{However, as we are not concerned with absolute frequency and magnitude identification in this work, we used DC motors due to their simplicity of use and low cost. The coding based on coupled vibration frequency and amplitude is simply referred to as magnitude modulation, as shown in Fig. 1.

The temporal sensitivity of the skin is very high, close to that of the auditory system and better than that of the visual system [22], which can be exploited in tactile patterns.
An example of temporal encoding is pulse train modulation, where short vibration bursts are a function of some physical value. For example, such mapping has been demonstrated for the purpose of prosthetic hand feedback [23], though pulse width modulation was reported to be inferior to amplitude modulation [24].

\subsection{Sensory integration of apparent motion speed and vibration magnitude}

Combining the spatial and temporal modulations can produce tactile apparent motion [18], [25], [26] as shown in the bottom of Fig. 1. Tactile apparent motion can provide an intuitive way of presenting spatial information, but discriminating between different motion speeds has been shown to be difficult [11]. We propose to address this issue by coupling the motion speed with vibration magnitude to emphasize the difference in speed and potentially improve the ability to discriminate between similar stimuli. The magnitude $(M)$, motion speed $(S)$ and our proposed modulation approach $(S \& M)$ are depicted in Fig. 1.

Fig. $2 \mathrm{~b}$ depicts the proposed coupling of motion speed and magnitude for the case of $L$ tactons. The $i$-th and $(i+$ $1)$ th tactons differ by the JND both in their speed of motion $S$, defined as the rotation frequency around the forearm, and the magnitude of vibrations $M$, controlled by motor voltage. This can be described using the Weber fraction as:

$$
\begin{aligned}
M_{i} & =M_{\max }\left(1+w_{M}\right)^{i-L} \\
S_{i} & =S_{\max }\left(1+w_{S}\right)^{i-L},
\end{aligned}
$$

where $w_{M}$ and $w_{S}$ are Weber fractions of magnitude and frequency mapping respectively, and $M_{\max }$ and $S_{\max }$ are the maximums at $i=L$. Inherently, the Stimulation Onset Asynchrony $\mathrm{SOA}_{i}=1 /\left(k S_{i}\right)$ (where $k$ is the array size) also changes according to the Weber's law. Fig. 2a illustrates the temporal and magnitude parameters for a three-actuator array. 


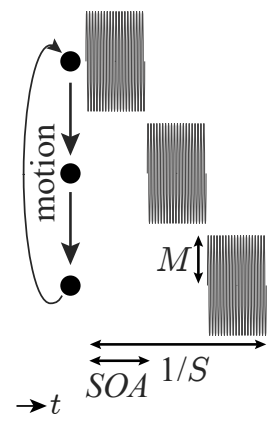

(a)

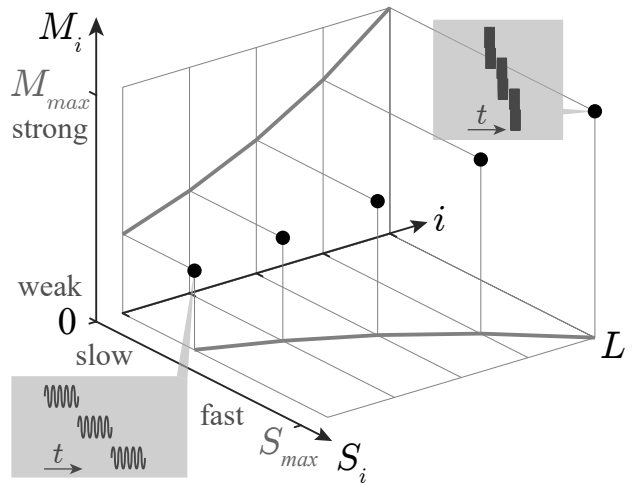

(b)

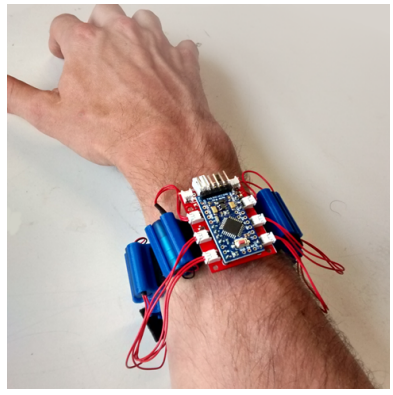

(c)

Fig. 2: Vibrotactile apparent motion. (a) Three motors in a closed loop array are sequentially activated to create continuous apparent motion. (b) The magnitude and stimulus frequency increase with $l$. The coupling results in stimuli ranging from slow $\mathcal{E}$ weak to fast $\mathcal{E}$ strong. (c) Photo of the tactile interface worn on the wrist.

As the changes of stimulus properties $S$ and $M$ are coupled, the delivery of information by the $S \& M$ stimulus is redundant. The nervous system seems to combine redundant sensory information in a fashion that is similar to a maximum-likelihood estimate (MLE), according to which the combined estimates should have lower variance, and therefore lower discrimination thresholds, than either estimate alone [12], [27]. Assuming this rule applies to the sensory integration of $S$ and $M$, we can express the detection threshold as Weber fraction of the coupled variable as

$$
\tilde{w}_{S \& M}^{2}=\frac{w_{S}^{2} w_{M}^{2}}{w_{S}^{2}+w_{M}^{2}} .
$$

Therefore we hypothesise that the Weber fraction of the $S \& M$ modulation will be smaller or at least equal to that of the individual $M$ and $S$ modulation.

\subsection{Apparent motion around the wrist}

Vibrotactile displays are commonly designed for stimulation of the torso [7], [28], [29] and the forearm [29]-[32]. Identification of the vibrotactile patterns can be superior on the torso [29], and the information display can be more intuitive in the case of navigation tasks due to the intuitive correspondence to the user's egocentric direction [6]. However, in tasks that include manual interaction, feedback displayed on the forearm is more intuitive. The wrist also offers the possibility of combining tactile feedback with co-located visual display and touch or gesture input. Such interactions are becoming increasingly interesting in the growing market of smart watches and similar personal wearable interfaces. Therefore, we chose to study our stimulation approach on the wrist.

In a preliminary study, we investigated the required actuator density to achieve a good illusion of circular motion around the forearm. In a forced-choice task the subjects were presented with stimuli induced by an array of either 3,4 or 6 motors and had to respond whether they feel clockwise or counter-clockwise motion. The results showed that three motors are not sufficient to yield a circular motion illusion, but the rate of correctly identified direction between four and six actuators was not statistically different. This result agrees with the study [33], that reported no significant advantage of using more than four actuators to display apparent motion around the upper arm. However, the subjective feedback from the users in our study revealed that six motors created a continuous sensation but four did not. Therefore we decided to use a six-actuator array in the present study.

Section 3 presents the evaluation of the highest perceivable apparent motion frequency around the forearm. Choosing fast apparent motions is key to achieve short tactons which in turn leads to shorter perception delays. Next, in section 4 we estimate the JND of $S \& M$ coding and compare it to the JND of the individual $S$ and $M$ coding. In section 5 we compile sets of tactons for $S \& M$, $M$ and $S$ codings following Eq. 1 . We examine the absolute discriminability of the tactons in a rating task and discuss the information transfer.

\section{EXPERIMENT 1: MAXIMAL SPEED OF MOTION}

First we evaluated the maximum motion speed at which the subjects were able to correctly identify the direction of motion. Based on the preliminary results we designed a wearable interface consisting of six actuators and evaluated the highest motion frequency that can be induced by such array. In a force choice task, the participants experienced apparent motion at different speeds and reported whether they felt a clockwise or anti-clockwise motion.

\subsection{Apparatus}

The wrist interface is shown in Fig. 2c. Shafted vibration motors were inserted into $3 \mathrm{D}$ printed housings to enable attachment to a velcro strap and provide sufficient contact area with the skin. Precision Microdrives model 307-100 (UK) motors were used, which have a rise time of $34 \mathrm{~ms}$ and a stop time of $73 \mathrm{~ms}$ (according to the data sheet). The motors were powered using haptic controllers DRV2603 (Texas Instruments, USA) which utilise feedback control to minimise the rise and stop times of a vibration motor by applying over-voltage and braking-voltage accordingly. The main module contains an Arduino microcontroller and haptic drivers, and communicated with the host PC via serial protocol. 


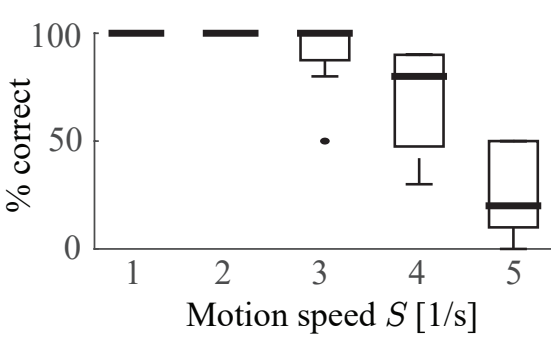

Fig. 3: Result of Experiment 1: distributions of correct answer percentage across subjects for the six wave frequencies.

\subsection{Methods}

Nine 21-30 years old subjects, recruited among graduate students and postdoctoral researchers at Imperial College, participated in the study. None of them reported any abnormalities in their tactile perception. All subjects had previous experience with vibrotactile stimulation. They were seated in front of a computer while wearing the device on the forearm of their right hand. To eliminate auditory cues from the motor noise, subjects wore noise cancellation headphones. Five different $S$ values were tested from 1 to 5 $s^{-1}$. There were 10 trials for each $S$ split between clockwise and counter-clockwise direction, resulting in 50 trials per subject. Each stimulus lasted two seconds. As we were only interested in the ability to identify the direction of motion, the magnitude of vibrations was kept constant; the voltage applied to the motors was equal to the nominal voltage of $3 \mathrm{~V}$ for any $S$. After each stimulus, the participants indicated whether they felt a clockwise or counter-clockwise motion around the forearm by clicking the respective buttons on the screen using a touchpad. The subjects were allowed to familiarise themselves with the stimuli before the experiment began.

\subsection{Results}

Fig. 3 shows the distribution of percentages of correctly identified direction of motion within subjects. For $S$ lower than $3 \mathrm{~s}^{-1}$, all subjects identified the direction of motion in $100 \%$ of trials. The majority of subjects were $100 \%$ correct in the case of $3 s^{-1}$, while the overall success rate across subjects was $90 \%$. In the case of $S=4 \mathrm{~s}^{-1}$, the overall success rate was reduced to $70 \%$.

\section{EXPERIMENT 2: SENSORY FUSION AND JND}

In this experiment we investigated the effects of combining the speed and magnitude modulations in the proposed apparent motion stimulus. We experimentally evaluated the Weber fraction of apparent motion speed $S$, vibration magnitude $M$ and the coupling $S \& M$. We fit psychometric functions to the experimental results and discuss the estimated Weber fractions with respect to the multimodal sensory integration concept summarised by Eq. 2 .

\subsection{Methods}

EXPERIMENTAL PROTOCOL. Ten subjects with age between 22 and 33 years old without any self reported abnormalities in tactile sensitivity took part in the force choice experiment.
They were recruited among researchers at Imperial College. All were familiar with vibrotactile stimulation and three subjects also participated in the previous experiment. The same experimental setup was used as in Experiment 1. The subjects sat at a desk facing a computer screen, used earplugs and noise cancellation headphones. They wore the interface on their right forearm and used the left hand to input the answers.

The method of constant stimuli [34] was used to determine the JND. In each trial, two stimuli of $500 \mathrm{~ms}$ were presented in succession separated by a $500 \mathrm{~ms}$ pause as in [16]. The subjects entered their answer same or different using a PC keyboard by pressing the $\mathrm{S}$ or D key, respectively. Between the trials, there was a pause of three seconds with the countdown shown on the screen, but the subjects could pause the countdown and rest at any time.

The experiment consisted of three phases, one for each modulation condition. Table 1 shows the experimental conditions for each block. Five $\triangle I / I$ values were tested from 0 to 0.4 for the case of low $I$ and high $I$, which were $33 \%$ and $66 \%$ of the nominal motor voltage $M_{\max }$, and $33 \%$ and $66 \%$ of the maximum $S_{\max }$ for $M$ and $\{S, S \& M\}$ modulations respectively. Each condition was repeated five times. There were 150 trials in total per subject, which lasted roughly 20-30 minutes to complete. In the case of the $M$ modulation, the actuator located on the volar side of the forearm produced tactile stimulation. We chose to use only one actuator instead of the entire array as in this way the mean stimulated area was equal for all modulations.

WEBER FRACTION ESTIMATION. The fitting of psychometric functions $p(x)$ allowed to predict the probability of perceiving a pair of stimuli as different for an arbitrary $x=\triangle I / I$ :

$$
p(x)=\left(1+\exp \left[-\frac{x-a}{b}\right]\right)^{-1}
$$

where $a$ shifts the psychometric function horizontally and represents the $50 \%$ detection threshold, while $b$ determines its slope [35]. We used the fit to estimate the Weber fractions of all three modulations.

\subsection{Results}

For each subject, the rates of stimuli perceived as different were computed for each condition (modulation, $I$ and $\triangle I / I$ ). Fig 4 shows the means and standard deviations of the rates between subjects. Participants identified the stimuli

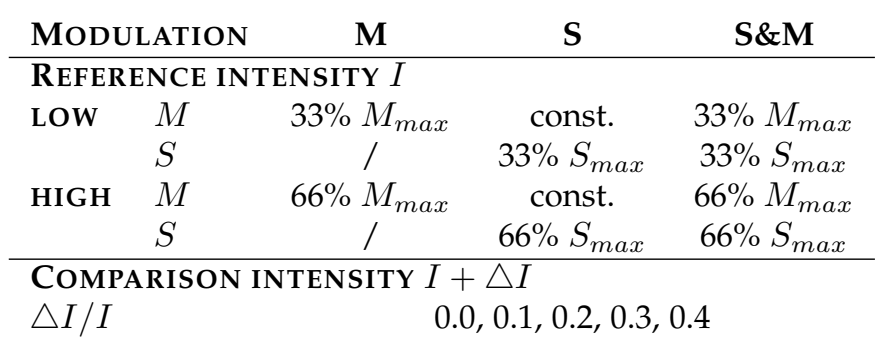

TABLE 1: Experiment 2 consisted of three blocks of trials, one for each modulation. In each block the JND was estimated around low and high $I . M_{\max }$ is the nominal motor voltage, and $S_{\max }$ is the maximal speed determined in Experiment 1. 

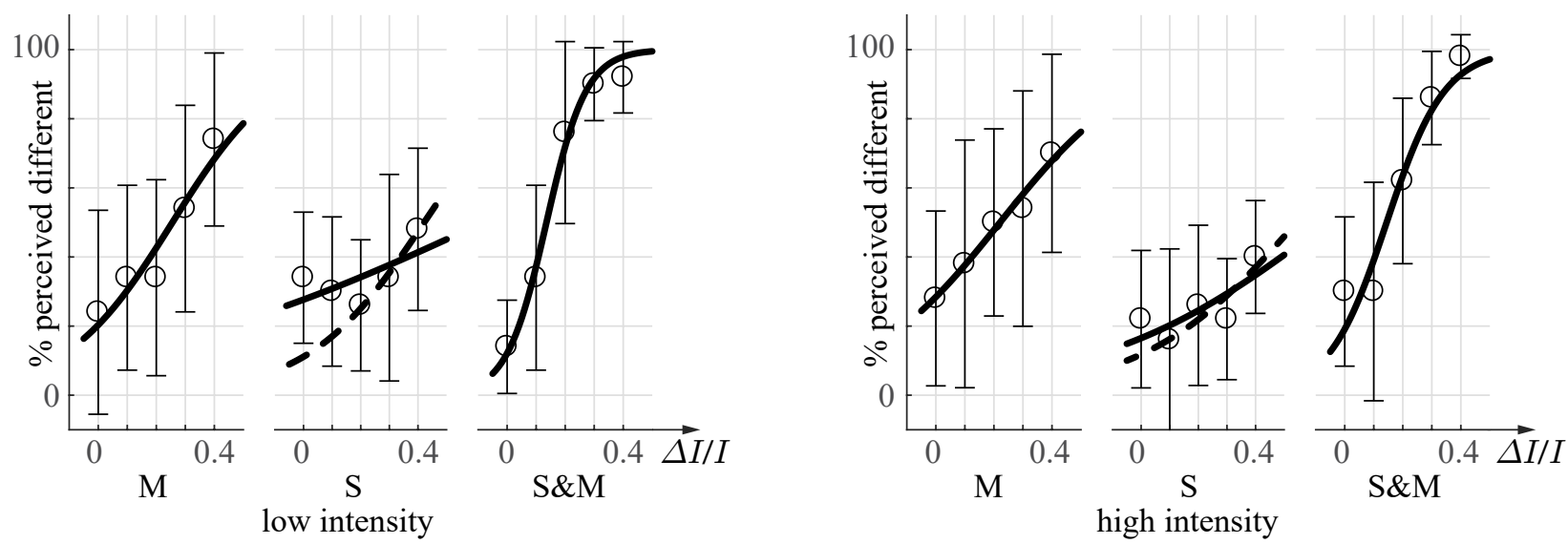

Fig. 4: Result of Experiment 2: Rates of stimuli perceived as different between subjects. Psychometric functions fitted to experimental data are overlaid. The dashed line represents the fits that only considered the data points for $\triangle I / I>0.1$.

as different in at least half of trials for $\triangle I / I \geq 0.3$ in case of $M$ (low $I$ ) and $\triangle I / I \geq 0.2$ for $S \& M$ (either $I$ ). In the case of $S$ modulation, the mean rate of stimuli perceived different never reached $50 \%$. Only three subjects identified the $S$ stimuli as different above the $50 \%$ threshold at $\triangle I / I=0.4$ for low and/or high $I$.

BETWEEN SUBJECT ANALYSIS OF RESPONSES. A 3-way (modulation, $I, \triangle I / I)$ ANOVA was performed which found no effect of low and high $I$ on the responses, nor any interaction of $I$ with other conditions. Further twosample tests were carried out for the effect of modulation on response rates per each $\triangle I / I$. For $\triangle I / I \geq 0.2$ the mean answer rate was found significantly higher for $S \& M$ w.r.t. the other two modulations ( $p<0.005$ in all 12 comparisons).

PSYCHOMETRIC FUNCTION AND JND. Psychometric functions defined by Eq. 3 were fit to the experimental data. The resulting curves are overlaid in Fig 4. Table 2 shows parameters $a$ which equal the $w=\frac{\triangle I_{\mathrm{IND}}}{I}$ where the curve crosses $50 \%$ rate, and the $R^{2}$ values which represent the quality of fit. Due to a large bias towards identifying small or zero $\triangle I / I$ as different in case of $S$ modulation, a second function was fit considering only points $\triangle I / I>0.1$. These fits are represented with dashed curves and $S^{*}$ column.

Based on the fits, the estimates of the Weber fractions could be obtained (see Table 2). The last two columns are the MLE estimates following Eq. 2.

\begin{tabular}{lllllll}
$I$ & $\mathbf{M}$ & \multicolumn{1}{c}{$\mathbf{S}$} & \multicolumn{1}{c}{$\mathbf{S}^{*}$} & $\mathbf{S} \& \mathbf{M}$ & MLE & MLE $^{*}$ \\
\hline LOW & 0.256 & 0.629 & 0.422 & 0.136 & 0.237 & 0.219 \\
& $(0.286)$ & $(0.041)$ & $(0.131)$ & $(0.743)$ & & \\
HIGH & 0.221 & 0.653 & 0.544 & 0.144 & 0.209 & 0.205 \\
& $(0.192)$ & $(0.084)$ & $(0.090)$ & $(0.617)$ & & \\
BOTH & 0.240 & 0.652 & 0.473 & 0.141 & 0.225 & 0.214 \\
& $(0.375)$ & $(0.105)$ & $(0.196)$ & $(0.759)$ & &
\end{tabular}

TABLE 2: Results of Experiment 2: Columns 2-5 show Weber fraction estimates derived from the psychometric function fits ( $R^{2}$ shown in brackets). Columns 6 and 7 show Weber fractions predicted by MLE.

\section{EXPERIMENT 3: TACTON IDENTIFICATION}

In general, absolute identification of stimuli is far more difficult than relative identification [3], [11], [36]. We compiled sets of tactons based on $M, S$ and $S \& M$ modulations and compared the participants' ability to discriminate between them.

\subsection{Methods}

For each modulation type $(M, S$ and $S \& M)$ a set of $L$ stimuli (tactons) and $L$ responses were compiled with oneto-one association. Participants were presented with the stimuli in random order. On each presentation, they picked a response. The collected responses were tabulated in the form of a stimulus-response confusion matrix.

TACTON SET. Given the previously estimated maximum speed of apparent motion and Weber fraction, an arbitrary number of tactons can be compiled. Enlarging the tacton set produces slower motion, which in turn requires longer time to complete the cycle around the forearm. Therefore, enlarging $L$ comes at the cost of longer tacton duration $T$. In this experiment, we choose $L$ such that the ratio $L / T$ is maximal.

$$
L / T=L f_{L}(1+w)^{1-L}
$$

Fig. 5 depicts $L / T$ (tactons/second) as a function of the number of levels $L$ for multiple values of the Weber fractions that we considered. Based on the results of the pre-

\begin{tabular}{|c|c|c|c|c|}
\hline block & $S \& M 5$ & $S \& M$ & $M$ & $S$ \\
\hline $\begin{array}{l}\text { CONDITIONS } \\
\text { modulation }\end{array}$ & $S \& M$ & $S \& M$ & $M$ & $S$ \\
\hline Weber fr./tactons & $0.2 / 5$ & $0.3 / 4$ & $0.3 / 4$ & $0.3 / 4$ \\
\hline tacton dur. T (ms) & 691 & 732 & 732 & 732 \\
\hline $\begin{array}{l}\text { TRIALS } \\
\text { exploring }\end{array}$ & \multicolumn{4}{|c|}{ variable (1-3 minutes) } \\
\hline practice & 10 & 8 & 8 & 8 \\
\hline testing & 40 & 32 & 32 & 32 \\
\hline
\end{tabular}

TABLE 3: Summary of experimental conditions. Four blocks tested different stimulation conditions resulting in 170 trials per subject. 
vious experiment where $w$ for both $M$ and $S \& M$ was $\leq 0.3$, we chose to compare all three modulations at 0.3 . From the analysis depicted in Fig. 5, the particular Weber fraction yields $L=4$. Additionally, $S \& M$ was also tested for $w=0.2$ and $L=5$ (condition $S \& M 5$ ). Tacton duration was chosen such that the slowest tacton completed one cycle. The conditions that characterise the tactons are listed in Table 3.

PARTICIPANTS AND PROTOCOL. Thirteen subjects between 23 and 34 years old, all researchers at Imperial College, participated in the study. None reported any abnormalities in their tactile perception. All subjects were familiar with vibrotactile stimulation. Although three of these subject also participated in the previous experiment, it is unlikely this prior experience gave them any advantage as several months passed between the experiments. The apparatus and experimental setup were as in previous experiments. At the beginning of the experiment, instructions were shown on the screen. The subjects were informed that the tactons may differ in speed and/or magnitude, but were not told what modulation was used in each of the four blocks. Table 3 describes the conditions of each block. The blocks are shown in the order as they appeared in the experiment. In each block, the test phase was preceded

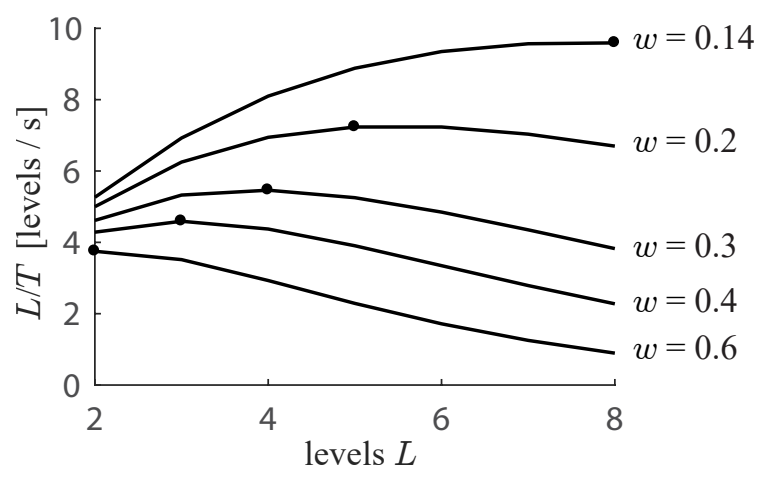

Fig. 5: Tactons per duration as functions of $L$ and their maxima (dots) for different Weber fraction values. The tick curves represent $w$ obtained from the same-different judgements, while the thin curves are for $w$ derived from fitted models.

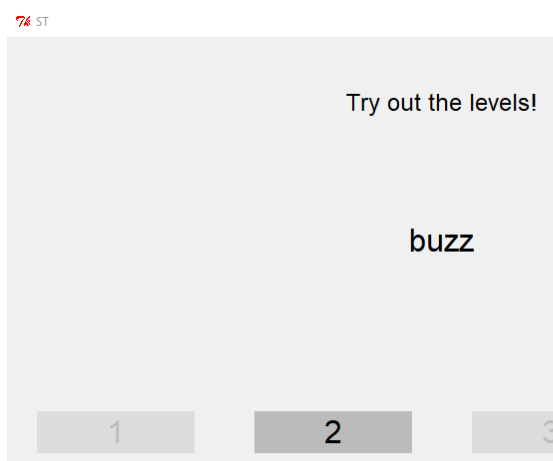

Fig. 6: The graphical interface in Experiment 3 during the familiarisation phase. The number two selected by the participant is highlighted. by the exploration and practice phase as in [11]. In the exploration phase the subjects first familiarized themselves with the tactons. By pressing the number keys ( 1 to $L$ ) on a standard computer keyboard they triggered the corresponding tacton. Their choice was also indicated on the computer screen (see Fig. 6 for the screen-shot of the graphic display). They explored different tactons until they felt they could distinguish between them and chose to conclude the exploration. Next was the practice phase, where the stimuli of all levels were presented in random order. Each condition was repeated twice and the stimulation was performed once without the possibility to repeat. The subjects rated the stimulus by pressing the corresponding number key. Their selection and the correct answer were displayed on the screen. In the test phase, the process was same, but the correct answer was not shown. Each condition was repeated 8 times during the test phase. At the end of the experiment the subjects were asked whether they realised the speed of motion was changing in the first two phases ( $S \& M 5$ and $S \& M)$.

DATA ANALYSIS. For each modulation we examined the rate of correctly identified stimulation levels (tactons) and the variance of error. Analysis of error is suitable for comparing results between equally sized sets of tactons. However, when comparing $S \& M, M$ and $S$ to $S \& M 5$, another method may provide a better interpretation of the results. Static information transfer rate quantifies the amount of information bits transferred to the user [37], [38]. It considers the distribution of error. The maximum likelihood estimate of data transfer $\beta$, derived from the stimulus-response matrix, is computed as

$$
\beta=\sum_{l=1}^{L} \sum_{m=1}^{L} \frac{n_{l m}}{n} \log _{2}\left(\frac{n_{l m} n}{n_{l} n_{m}}\right),
$$

where $n$ is the total number of trials, $n_{l m}$ the number of correct responses, $n_{l}$ and $n_{m}$ are the row and column sums [37].

\subsection{Results}

CONFUSION MATRICES. The number of correct answers was compared over all subjects. The stimulus-response confusion matrices are shown in Fig. 7. For the case of $L=4$, we can compare the overall rate of correct answers for all three modulations. Subjects performed best in the case of $S \& M$ with $71 \%$ correct compared to $66 \%$ and $34 \%$ for the case of $M$ and $S$ respectively. Four of the 13 subjects reported that they did not realise the speed of motion was varied in the $S \& M$ blocks. In average these four subject identified $M$ tactons more accurately than $S \& M$ tactons (69\% versus $57 \%$ ), but performed better in $S \& M$ than $S(57 \%$ versus $28 \%)$.

AtTEnTION TO MOTION SPEED. Based on the observation that four subjects did not detect speed variations in $S \& M$ and $S \& M 5$ trial blocks, we examined the effect of this self-reported factor that we call awareness. A twoway ANOVA was performed to test for the interaction between the conditions block and awareness. Significant effects were found for both conditions and their interaction $(p<0.005, p<0.005$ and $p<0.020)$. Follow-up multiple 


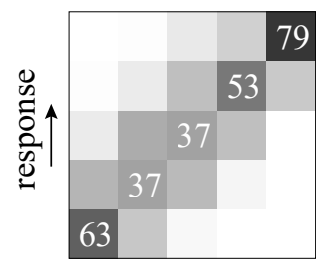

total correct: $53 \%$ block: $\quad$ S\&M5

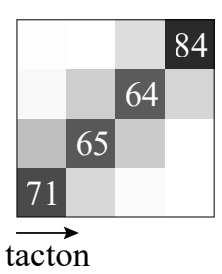

$71 \%$

$\mathrm{S} \& \mathrm{M}$

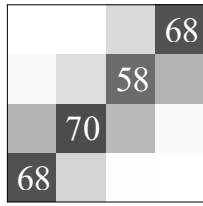

$66 \%$

$\mathrm{M}$

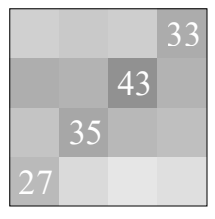

$34 \%$

$\mathrm{S}$

(a) stimulus-response confusion matrices
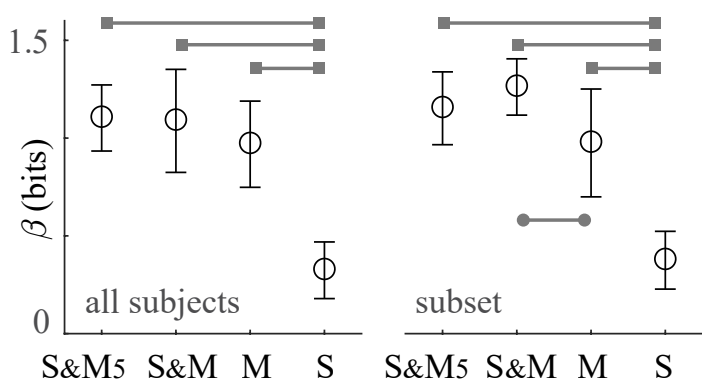

(b) information transfer between subject

Fig. 7: Result of Experiment 3: (a) Confusion matrices depict the frequency of responses in \% across all subjects. (b) Distributions of static information transfer rate between all (left) and the 9-subject subset (right).

comparison tests confirmed the interaction in case of the $S \& M$ block $(p<0.012)$, but not in others $(p \geq 0.55)$.

INFORMATION TRANSFER. The results of information transfer estimation $\beta$ computed using Eq. 5 are shown in Fig. $7 \mathrm{~b}$ for all subjects (left) and the subjects aware of speed changes (subset; right). One-way ANOVA tests confirmed the effect of modulation on $\beta$ for both subject sets. Further two-sample T-tests were carried out to test which differences in means of $\beta$ are significantly different. The horizontal lines link the significantly different groups. In the case of circle arrowheads the significance was $p<0.031$, whereas the square arrowheads denote $p<0.005$ significance.

TIME TO ANSWER. Between subjects mean time required to answer was $2.21,1.62,1.47$ and 1.82 seconds for $S \& M 5$, $S \& M, M$ and $S$ respectively. Pairwise two-sample T-tests showed the mean time for $S \& M 5$ was significantly longer than $S \& M$ and $M(p<0.01)$.

\section{Discussion}

In this work we presented a concept of design of tactons based on apparent motion. To achieve fast information transfer, we aimed to design short yet distinct tactons. We developed a tactile interface that creates vibrotactile apparent motion around the forearm. To improve the ability to discriminate between motions of different speeds, we proposed to couple the speed with vibration magnitude, resulting in stimuli that range from slow and weak to fast and strong. We evaluated the fastest perceivable motion speed and investigated the relative and absolute discrimination of the individual and coupled modulations by estimating the JND and information transfer.

MAXIMAL SPEED AND HARDWARE LIMITATIONS. We found that most subjects were able to perceive the direction of the motion correctly at 3 rotations per second. At $S=4 s^{-1}$ the SOA equals $41.7 \mathrm{~ms}$, but humans can detect vibration pulses and gaps of $10 \mathrm{~ms}$ [39], [40]. The said SOA is very close to the rise time of the actuator used in this work, therefore we conclude that the ability to perceive the direction of motion correctly was limited by the motor dynamics, rather than by the human tactile perception. Higher motion speed may be achievable using voice coils (linear actuators), such as the Haptuator (Tactile Labs, USA). These are typically quicker and offer independent control of vibration frequency and amplitude, but the cost of these actuators is considerably higher, and the actuators require more complex AC driver circuits.

JND OF MAGNITUDE AND APPARENT MOTION SPEED. By fitting the psychometric function we obtained an estimate of Weber fractions for each modulation. $w$ for $M$ modulation was estimated between 0.22 and 0.26 , which agrees with [14]. We found that in the case of $S$ modulation it is very difficult to discriminate between similar motion speeds even for $\triangle I / I$ as high as 0.4 . By fitting psychometric functions to the experimental results, we were able to obtain estimates of Weber fraction between 0.422 and 0.629 . These estimates are higher than the results in [11], which reported $w$ of 0.25 . However, in the said study the stimuli were six times longer (lasting 3 seconds), which could explain the difference as the participants were given more time to sample the motion speed during each stimulus presentation. Thus, a further evaluation of JND at different stimulus durations is required.

MLE SENSORY INTEGRATION. The comparison of answer rates and the estimated JND show superior performance of the coupled $S \& M$ modulation, which confirms our hypothesis that the discrimination ability of the coupled variable is equal or better than that of the individual variables. However, the detection thresholds for the coupled modulation predicted by the MLE (Eq. 2) shown in Table 2 are substantially larger than those experimentally obtained. Explaining this result with probability summation was also dismissed, as it could only explain the case where the detection threshold of the coupled variable ranks between those of individual ones. Perhaps this difference between the predicted and measured threshold is caused by the tactile spatial summation phenomenon [41]; although the total stimulated area of the skin was constant and equal for all three modulations, in case of $M$ the total number of stimulated tactile receptors during the stimulus duration was smaller as only one actuator was used. Therefore, future experiments should compare the JND of magnitude changes for the case of $M$ modulation and $S \& M$ modulation with constant motion speed.

ABSOLUTE DISCRIMINATION AND INFORMATION TRANSFER. Between-subject analysis showed that compared to $M$ and $S, S \& M$ enabled more correct answers and higher information transfer $\beta$. Although the estimates of $\beta$ for $S \& M$ and $S \& M 5$ (see Fig. $7 \mathrm{~b}$ ) were not substantially higher than those obtained in [11] for apparent motion (1.02 
bits), the tacton duration in our study was roughly four times shorter. Therefore, thanks to coupling of the sensory cues, our approach allows to achieve higher $\beta$ values for much shorter tactons and thus higher information transfer rate $\beta / T$ (bits/second). The within-subjects average time to answer was smallest in case of $M$ modulation, followed by $S \& M$ with $9 \%$ longer reaction times, however, no significant difference was found between these two. Finally, as the subjects were not explicitly told what cues to pay attention to, four of them did not realise that the speed was changing in case of $S \& M$. These subjects performed best in $M$ tacton identification as they did not pay attention to speed changes and thus could not take advantage of redundant cue coupling. It remains unclear whether instructing them explicitly would yield better performance, but prolonging the tacton duration may enable them to realise the coupling during training and facilitate better identification performance.

To summarise, the results show that the relative discrimination of the coupled variable can be characterised by JND smaller than JNDs of the individual variables. This allowed us to create short tactons for display of direction and speed, which can be distinguished significantly better than tactons based on motion alone. Most subjects were also able to identify the coupled-variable tactons better than the magnitudebased tactons. As our experiments were conducted using stimulation of the wrist, the results are directly applicable to personal wearable devices such as smart watches. The proposed coupled modulation could for example be employed to render different angles of a turn in spatial navigation tasks. Additionally, the coupled variable could present the priority of an incoming message, while the direction of motion could suggest the context (work or private message). Thanks to the lower JND of the proposed coupled variable, this feedback approach could also be employed for real time feedback of vector quantities in manual interactions. For instance, the coupled variable could encode the torque exerted on the wrist of a teleoperated robot. However, the results highlight the need for further studies to investigate the dependency of apparent motion perception on stimulus duration, the potential effect of spatial summation on the perception of magnitude of $S \& M$ stimuli, and the role of user's attention to motion speed in absolute identification.

\section{ACKNOWLEDGEMENT}

This work is supported by the European Commission under EU-FP7 grants PEOPLE ITN-GA-2012-317488-CONTEST, ICT-601003 BALANCE, ICT-2013-10 SYMBITRON and H2020 ICT 644727 COGIMON.

\section{REFERENCES}

[1] K. MacLean and M. Enriquez, "Perceptual design of haptic icons," in Proc. of EuroHaptics, 2003, pp. 351-363.

[2] J. B. van Erp and M. M. Spapé, "Distilling the underlying dimensions of tactile melodies," in Proceedings of Eurohaptics, vol. 2003, 2003, pp. 111-120.

[3] S. Brewster and L. M. Brown, "Tactons: structured tactile messages for non-visual information display," in Proceedings of the fifth conference on Australasian user interface-Volume 28. Australian Computer Society, Inc., 2004, pp. 15-23.
[4] D. Ternes and K. E. Maclean, "Designing large sets of haptic icons with rhythm," in International Conference on Human Haptic Sensing and Touch Enabled Computer Applications. Springer, 2008, pp. 199208.

[5] L. Brunet, C. Megard, S. Paneels, G. Changeon, J. Lozada, M. P. Daniel, and F. Darses, "invitation to the voyage: The design of tactile metaphors to fulfill occasional travelers' needs in transportation networks," in World Haptics Conference (WHC), 2013. IEEE, 2013, pp. 259-264.

[6] D. J. Barber, L. E. Reinerman-Jones, and G. Matthews, "Toward a tactile language for human-robot interaction: Two studies of tacton learning and performance," Human factors, vol. 57, no. 3, pp. 471-490, 2015.

[7] J. B. Van Erp, H. A. Van Veen, C. Jansen, and T. Dobbins, “Waypoint navigation with a vibrotactile waist belt," ACM Transactions on Applied Perception (TAP), vol. 2, no. 2, pp. 106-117, 2005.

[8] C. Ho, H. Z. Tan, and C. Spence, "Using spatial vibrotactile cues to direct visual attention in driving scenes," Transportation Research Part F: Traffic Psychology and Behaviour, vol. 8, no. 6, pp. 397-412, 2005.

[9] M. Ogrinc, I. Farkhatdinov, R. Walker, and E. Burdet, "Horseback riding therapy for a deaf-blind individual enabled by a haptic interface," Assistive Technology, 2017.

[10] — " "Deaf-blind can practise horse riding with the help of haptics," in Haptics: Perception, Devices, Control, and Applications: 10th International Conference, EuroHaptics 2016, London, UK, July 47, 2016, Proceedings. Springer, 2016, pp. 452-461.

[11] L. Kohli, M. Niwa, H. Noma, K. Susami, Y. Yanagida, R. W. Lindeman, K. Hosaka, and Y. Kume, "Towards effective information display using vibrotactile apparent motion," in Haptic Interfaces for Virtual Environment and Teleoperator Systems, 2006 14th Symposium on. IEEE, 2006, pp. 445-451.

[12] M. O. Ernst and M. S. Banks, "Humans integrate visual and haptic information in a statistically optimal fashion," Nature, vol. 415, no. 6870, pp. 429-433, 2002.

[13] G. A. Gescheider, Psychophysics: the fundamentals. Psychology Press, 2013.

[14] S. Choi and K. J. Kuchenbecker, "Vibrotactile display: Perception, technology, and applications," Proceedings of the IEEE, vol. 101, no. 9, pp. 2093-2104, 2013.

[15] E. Francisco, V. Tannan, Z. Zhang, J. Holden, and M. Tommerdahl, "Vibrotactile amplitude discrimination capacity parallels magnitude changes in somatosensory cortex and follows webers law," Experimental brain research, vol. 191, no. 1, p. 49, 2008.

[16] H. Pongrac, "Vibrotactile perception: Differential effects of frequency, amplitude, and acceleration," in Haptic Audio Visual Environments and their Applications, 2006. HAVE 2006. IEEE International Workshop on. IEEE, 2006, pp. 54-59.

[17] D. A. Mahns, N. Perkins, V. Sahai, L. Robinson, and M. Rowe, "Vibrotactile frequency discrimination in human hairy skin," Journal of neurophysiology, vol. 95, no. 3, pp. 1442-1450, 2006.

[18] J. H. Kirman, "Tactile apparent movement: The effects of interstimulus onset interval and stimulus duration," Perception $\mathcal{E}$ Psychophysics, vol. 15, no. 1, pp. 1-6, 1974.

[19] F. W. Nutter and P. D. Esker, "The role of psychophysics in phytopathology: The weber-fechner law revisited," European Journal of Plant Pathology, vol. 114, no. 2, pp. 199-213, 2006.

[20] I. Hwang, J. Seo, M. Kim, and S. Choi, "Vibrotactile perceived intensity for mobile devices as a function of direction, amplitude, and frequency," Haptics, IEEE Transactions on, vol. 6, no. 3, pp. 352-362, 2013.

[21] J. Ryu, J. Jung, G. Park, and S. Choi, "Psychophysical model for vibrotactile rendering in mobile devices," Presence: Teleoperators and Virtual Environments, vol. 19, no. 4, pp. 364-387, 2010.

[22] L. A. Jones and S. J. Lederman, Human hand function. Oxford University Press, 2006.

[23] A. Chatterjee, P. Chaubey, J. Martin, and N. Thakor, "Testing a prosthetic haptic feedback simulator with an interactive force matching task," JPO: Journal of Prosthetics and Orthotics, vol. 20, no. 2, pp. 27-34, 2008.

[24] C. E. Stepp and Y. Matsuoka, "Vibrotactile sensory substitution for object manipulation: amplitude versus pulse train frequency modulation," Neural Systems and Rehabilitation Engineering, IEEE Transactions on, vol. 20, no. 1, pp. 31-37, 2012.

[25] T. Tedesco, A. Feigl, and H. Genet, "The cutaneous" rabbit": a perceptual illusion," 1972. 
[26] A. Ninu, S. Dosen, D. Farina, F. Rattay, and H. Dietl, "A novel wearable vibro-tactile haptic device," in Consumer Electronics (ICCE), 2013 IEEE International Conference on. IEEE, 2013, pp. $51-52$.

[27] M. O. Ernst and H. H. Bülthoff, "Merging the senses into a robust percept," Trends in cognitive sciences, vol. 8, no. 4, pp. 162-169, 2004.

[28] F. Hao and A.-g. Song, "Design of a wearable vibrotactile display waist belt," in Intelligent Control and Automation (WCICA), 2010 8th World Congress on. IEEE, 2010, pp. 5014-5017.

[29] E. Piateski and L. Jones, "Vibrotactile pattern recognition on the arm and torso," in Eurohaptics Conference, 2005 and Symposium on Haptic Interfaces for Virtual Environment and Teleoperator Systems, 2005. World Haptics 2005. First Joint. IEEE, 2005, pp. 90-95.

[30] B. Weber, S. Schätzle, T. Hulin, C. Preusche, and B. Deml, "Evaluation of a vibrotactile feedback device for spatial guidance," in World Haptics Conference (WHC), 2011 IEEE. IEEE, 2011, pp. 349354.

[31] I. Oakley, Y. Kim, J. Lee, and J. Ryu, "Determining the feasibility of forearm mounted vibrotactile displays," in Haptic Interfaces for Virtual Environment and Teleoperator Systems, 2006 14th Symposium on. IEEE, 2006, pp. 27-34.

[32] H.-Y. Chen, J. Santos, M. Graves, K. Kim, and H. Tan, "Tactor localization at the wrist," Haptics: Perception, Devices and Scenarios, pp. 209-218, 2008.

[33] M. Niwa, R. W. Lindeman, Y. Itoh, and F. Kishino, "Determining appropriate parameters to elicit linear and circular apparent motion using vibrotactile cues," in EuroHaptics conference, 2009 and Symposium on Haptic Interfaces for Virtual Environment and Teleoperator Systems. World Haptics 2009. Third Joint. IEEE, 2009, pp. 75-78.

[34] D. I. Shore, K. Gray, E. Spry, and C. Spence, "Spatial modulation of tactile temporal-order judgments," Perception, vol. 34, no. 10, pp. 1251-1262, 2005.

[35] F. A. Wichmann and N. J. Hill, "The psychometric function: I. fitting, sampling, and goodness of fit," Attention, Perception, $\mathcal{E}$ Psychophysics, vol. 63, no. 8, pp. 1293-1313, 2001.

[36] F. A. Geldard, "Some neglected possibilities of communication," Science, vol. 131, no. 3413, pp. 1583-1588, 1960.

[37] H. Z. Tan, C. M. Reed, and N. I. Durlach, “Optimum information transfer rates for communication through haptic and other sensory modalities," IEEE Transactions on Haptics, vol. 3, no. 2, pp. 98-108, 2010.

[38] W. R. Garner, "Uncertainty and structure as psychological concepts." 1962.

[39] L. Petrosino and D. Fucci, "Temporal resolution of the aging tactile sensory system," Perceptual and motor skills, vol. 68, no. 1, pp. 288290, 1989.

[40] G. Gescheider, S. Bolanowski, and R. Verrillo, "Temporal relations in cutaneous stimulation," Cutaneous communication systems and devices, pp. 33-37, 1974.

[41] G. A. Gescheider, S. J. Bolanowski, J. V. Pope, and R. T. Verrillo, "A four-channel analysis of the tactile sensitivity of the fingertip: frequency selectivity, spatial summation, and temporal summation," Somatosensory \& motor research, vol. 19, no. 2, pp. 114-124, 2002.

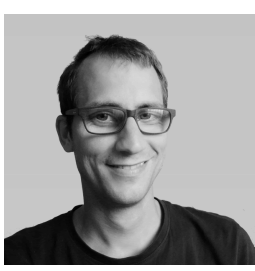

Matjaž Ogrinc is a doctoral candidate at Imperial College London with the Human Robotics Group and a research associate at Shadow Robot Company. He joined Imperial and Shadow as a Marie Curie fellow in August 2013. He got his Master of Research degree in Bioengineering with distinction from Imperial in October 2014 and his University Diploma in Electrical Engineering from University of Ljubljana (Slovenia) in November 2012. Between January 2011 and November 2012, Matjaž was a researcher with Humanoid and Cognitive Robotics Lab at Jožef Stefan Institute (Ljubljana, Slovenia).

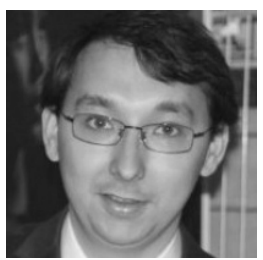

Dr Ildar Farkhatdinov is a Lecturer in Robotics at the School of Electrical Engineering and Computer Science at Queen Mary University of London (QMUL) and an Honorary Lecturer at the Department of Bioengineering of Imperial College London. Before joining QMUL he was a research associate at Imperial College of London. He got his Ph.D. in Robotics from University Pierre and Marie Curie, Paris VI Sorbonne (Paris, France), M.Sc. in Mechanical Engineering from Korea University of Technology and Education (Cheonan, South Korea), and B.Sc. in Automation and Control from Moscow State University of Technology STANKIN (Moscow, Russia).

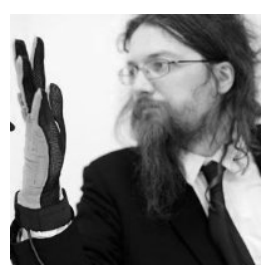

Rich Walker has spent years working in robotics and now leads the team at Shadow who are developing new robots and new applications for robotics. He obtained his Diploma in Computer Science in 1993 and BA in Mathematics in 1992 from University of Cambridge.

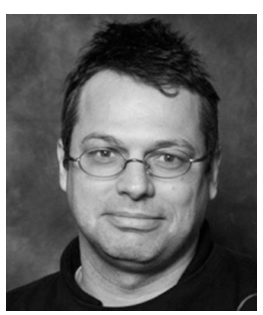

Prof Etienne Burdet is Professor of Human Robotics in the Department of Bioengineering at Imperial College London and visiting Professor at Nanyang Technological University and at UCL. He obtained an MSc in Mathematics (1990), MSc in Physics (1991), and PhD in Robotics (1996), all from ETH-Zurich. Etienne was a postdoctoral fellow with Theodore Milner from McGill University, Canada, J Edward Colgate from Northwestern University, USA and Mitsuo Kawato of ATR in Japan. He is pursuing research in robotics and bioengineering, where his main interest is in human machine interaction. 